\title{
Differential conditioning of conditioned enhancement and positive conditioned suppression
}

\author{
DONALD MELTZER \\ Southern Illinois University, Carbondale, Illinois 62901 \\ and \\ ROBERT J. HAMM \\ Virginia Commonwealth University, Richmond, Virginia 23220
}

\begin{abstract}
Rats, barpressirig for sucrose solution, were divided into three groups, each of which was exposed to a different VI schedule. During each session, four long tone CSs and four short light CSs were followed by a food pellet for half of each group. For the other half, the long CS was a tone and the short CS was light. Response rate increased during long CSs and decreased during short CSs regardless of the type of stimulus. It was concluded that conditioned enhancement and positive conditioned suppression could be brought under stimulus control and that the different phenomena were a function of CS duration.
\end{abstract}

Several years ago Meltzer and Brahlek (1970) showed that rats which received food for pressing a bar would sometimes increase and sometimes decrease their response rate during a conditioned stimulus (CS). The CS preceded presentations of a positive unconditioned stimulus (UCS), a sucrose solution which was not contingent on responding. Whether the subject's response rate increased or decreased depended upon the duration of the CS: If a 2-min stimulus was used, rate increased; if a stimulus of less than $40 \mathrm{sec}$ was used, rate decreased. The former result was called conditioned enhancement; the latter, positive conditioned suppression. Other studies using rats have found either conditioned enhancement during a long CS (Brady, 1961; Meltzer \& Hamm, 1974a, 1974b) or positive conditioned suppression during a short CS (Azrin \& Hake, 1969; Hake \& Powell, 1970; Van Dyne, 1971), but have not reported both effects in the same experiment. Moreover, Mackintosh (1974) recently raised a question about the validity of the conditioned enhancement effect. He argued that since Meltzer and Brahlek (1970) presented long and short CSs in different blocks of sessions, conditioned enhancement was actually caused by a decrease in baseline rate over the course of the experiment rather than by a real increase in rate during the $\mathrm{CS}$. The present experiment was performed to show that increases and decreases in CS response rate as a function of CS duration could be brought under stimulus control during a single session. That is, rats would show conditioned enhancement during a stimulus associated with a long CS and positive conditioned suppression during a stimulus associated with a short CS. Thus, neither effect could be explained as the result of different baseline response rates.
At the same time, several other questions were examined. Meltzer and Hamm (1974a) reported that rats showed greater conditioned enhancement when the baseline schedule was variable interval (VI) $4 \mathrm{~min}$ as opposed to VI $30 \mathrm{sec}$. Therefore, different schedules were used in this experiment to see if the schedule affected the relative magnitude of response rate changes during the CS.

In addition, Hearst and Jenkins' (1974) explanation of why response rate decreased during short duration CSs was also examined. They said that positive conditioned suppression was a consequence of sign tracking, which they defined as "behavior that is directed toward or away from a stimulus as a result of the relation between that stimulus and the reinforcer" (p. 4). The CS in most of those experiments in which positive conditioned suppression was produced, was an easily localized stimulus such as a cue light. If rats approached, or oriented toward, the cue light, the sign tracking behavior would interfere with barpressing and cause the observed suppression. That theory suggests that conditioned enhancement should be established most easily in rats when the long CS is a diffuse stimulus, such as a tone, and the short CS is a localized stimulus, such as a cue light. That relationship between CS duration and type of stimulus was expected to lead to good discrimination in the present experiment, while using tone as the short CS and light as the long CS was expected to lead to poorer discrimination.

\section{METHOD}

\section{Subjects}

Eighteen male hooded rats, approximately 90 days old 
at the beginning of the experiment, were used. Ten days prior to the beginning of the experiment, subjects were placed on a 23-h food-deprivation schedule. During the experiment each subject was given free access to food in its home cage for $1 \mathrm{~h}$ each day. Water was continuously available in each subject's cage. One animal died during the course of the experiment.

\section{Apparatus}

Rats were tested in six standard operant conditioning chambers equipped with a bar, pellet dispenser, liquid dipper, cue light, and sonalert audio signal. A complete description was provided in Meltzer and Hamm (1974b). White noise was always present in the experimental room to mask extraneous noises. Electromechanical relay equipment located in an adjacent control room controlled stimulus events and recorded responses.

\section{Procedure}

The bars were removed from the chambers for the first three sessions of the experiment. During the first two sessions, the liquid dipper delivered a $10 \%$ sucrose solution (by weight) on a variable-time (VT) 1-min schedule. Sessions lasted for $50 \mathrm{~min}$, and all subjects drank from the dipper consistently by the end of the second session. During the third session, a 45-mg Noyes pellet was delivered to the food hopper on a VT 1-min schedule. By the end of the session, all subjects ate each food pellet immediately upon delivery.

The bars were placed in the chambers at the beginning of Session 4 and remained in place for the rest of the experiment. During Sessions 4 and 5, barpressing was reinforced with $6 \mathrm{sec}$ of access to the sucrose solution on a VI $30-\mathrm{sec}$ schedule. The schedule was raised to VI $1 \mathrm{~min}$ for the next five sessions. Starting with Session 11, subjects were divided into three groups of six rats each. Responses were reinforced with sucrose on a VI 2-min schedule for subjects in Group 1, on a VI 4-min schedule for subjects in Group 2, and on a VI 8 -min schedule for subjects in Group 3. These schedules were maintained to the end of the experiment.

CS-UCS pairings were introduced during Session 11. Each subject received eight trials per session in which a CS preceded the $45-\mathrm{mg}$ food-pellet UCS. The CS remained on for $2 \mathrm{~min}$ prior to the UCS in four of the trials and for $20 \mathrm{sec}$ in the other four trials. For three subjects in each group, the longduration CS consisted of turning on the cue light mounted on the front wall of the chamber. The short CS was the tone produced by the sonalert mounted on the back wall of the chamber. The stimuli which were used as the long and short CSs were reversed for the other three subjects in each group. This procedure continued for the remaining 40 experimental sessions.

Total responses and total reinforcements were recorded in each of the last 40 sessions. In addition, responses during the four 2-min intervals which preceded long CSs were recorded, and the number of responses during the 1 st and 2 nd min of the four 2-min CSs were also recorded. A similar procedure was used when short CSs were presented. The number of responses during the four 20-sec intervals which preceded each short CS were recorded, as were the number of responses during the first and second $10 \mathrm{sec}$ of the four short CSs.

Inflection ratios were calculated for each subject's performance during the long and short CSs for each session, as described in Meltzer and Hamm (1974a). Mean inflection ratios for both long and short CSs were then calculated for each subject in successive four-session blocks. An inflection ratio above .5 meant that rate increased during the $\mathrm{CS}$; an inflection ratio below .5 meant rate had decreased during the CS. The mean pre-CS response rates and mean response rates during both the first and second halves of the CS were also calculated for each subject in four-session blocks.

Finally, one other question was examined. Although conditioned enhancement has been shown repeatedly in the rat, it has been demonstrated only during long CSs. It seemed possible that the difference in rate changes during long and short CSs might be accounted for by very simple temporal parameters. For example, all subjects might show a suppression of response rate during the last few seconds of the CS before the UCS. But with a 2 -min CS, the increase in rate during the early part of the CS might be large enough to produce an overall enhancement of mean response rate. It is also possible that a transient suppression of rate might occur during the first few seconds after the onset of a stimulus preceding the UCS. In that case, an increase in rate during the latter part of a long CS would produce the conditioned enhancement. Thus, unless response rates are shown to be higher during both the beginning and end of long CSs than during similar portions of short CSs, there is no reason to suppose that conditioned enhancement and positive conditioned suppression represent real differences in CS rates. The observed differences could be explained as a consequence of the fact that measurements are taken during different parts of the CS-UCS interval. During the last block of sessions of this experiment, all previous measures, as well as the number of responses made during the first and last $20 \mathrm{sec}$ of the 2-min CS, were recorded

Two separate sets of analyses were performed on the data. Inflection ratios and response rates from the first five blocks of sessions were analyzed using standard factorial procedures. However, since one subject in the VI 4-min group became ill during the sixth block of sessions, the last five blocks were analyzed using a design for unequal and nonproportional cell frequencies (Myers, 1972, pp. 114-121).

\section{RESULTS}

Figure 1 shows the mean inflection ratio of all subjects during the long and short CSs over the 10 blocks of sessions. The ratio was considerably higher during the 2-min CS than during the 20-sec CS. This difference was highly significant during both the first five blocks of sessions $(F=33.58, \mathrm{df}=1 / 12, \mathrm{p}<.01)$

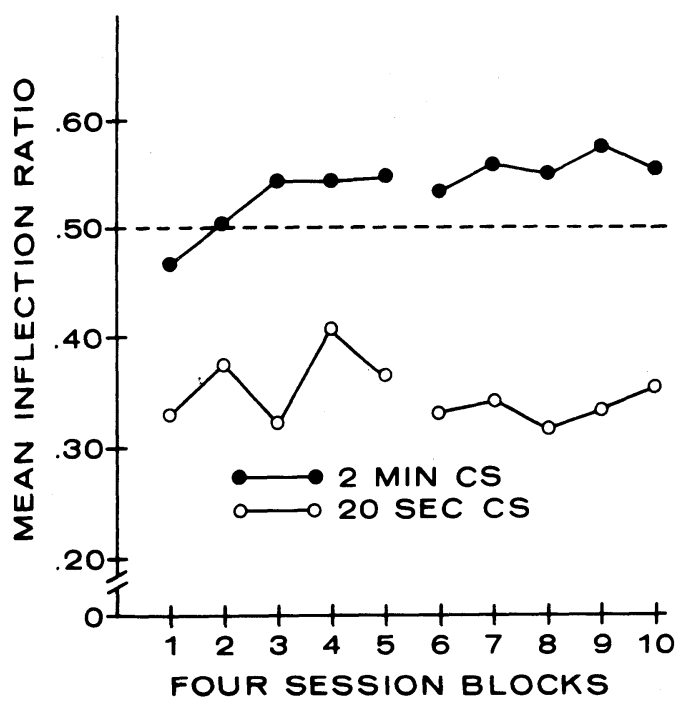

Figure 1. Mean inflection ratios during the long and short CSs in each of the 10 blocks of sessions. All points above .5 indicate conditioned enhancement; all points below .5 indicate conditioned suppression. 
and the last five blocks of sessions $(F=198.80$, $\mathrm{df}=1 / 11, \mathrm{p}<.001)$.

Perhaps a more important question is whether the mean inflection ratio was significantly above .5 during the long CS and significantly below .5 during the short CS. Data from the first two blocks of sessions were not examined since inflection ratios were changing rapidly during the long $\mathrm{CS}$ at this time. During the third, fourth, and fifth blocks of sessions, 16,13, and 12 , respectively, of the 18 subjects had inflection ratios above .5. Of the 17 subjects in Blocks 6-10, 13, $16,13,16$, and 16 , respectively, had inflection ratios above .5 . A binomial test revealed that the probability that 12 of 18 subjects would be above .5 by chance is less than .05 ; the probability that 13 of 17 subjects would be above .5 by chance is less than .01 . During the short CS, either 12 or 13 subjects always had inflection ratios below .5 in every block of sessions. The probability that 12 of 18 subjects would be below .5 by chance is less than .05 . The probability that 12 of 17 subjects would be below .5 by chance is less than .025 .

Inflection ratios during the first five blocks of sessions were unaffected by the schedule of reinforcement, and there were no significant interactions in which schedule was a factor.

During the last five blocks of sessions, the kind of stimulus which served as the long or short CS also affected the ratio. For those subjects for which light was the 2-min CS and tone the 20-sec CS, the mean inflection ratios across all five blocks were .538 and .442 , respectively. For those subjects for which the tone was the 2-min CS and light the 20-sec CS, the inflection ratios were .571 and .239 , respectively. In short, greater enhancement and stronger suppression were produced when tone was the long CS and light the short CS than when the stimulus conditions were reversed $(F=60.51, \mathrm{df}=1 / 11, \mathrm{p}<.001)$.

Another set of analyses was performed on response rates during the two sets of sessions. As in the inflection ratio analyses, the data from the first five blocks of sessions were analyzed using a standard factorial design and the data from the last five blocks of sessions were analyzed using a design for unequal and nonproportional cell frequencies. Response rates during both the pre-CS and CS intervals are shown in Figure 2. The generally increasing trend of response rates during the first five blocks of sessions was significant $(F=5.81$, $\mathrm{df}=4 / 48, \mathrm{p}<.05$ ), but no significant interactions were found in which blocks was a factor. Response rate did not change significantly across the last five blocks of sessions. Table 1 shows mean response rates during both the pre-CS and CS intervals for each group exposed to the different schedules in the first and last five blocks. The difference between schedules was significant during the first five blocks $(F=3.94$, $\mathrm{df}=2 / 12, \mathrm{p}<.05)$ but not during the last five blocks.

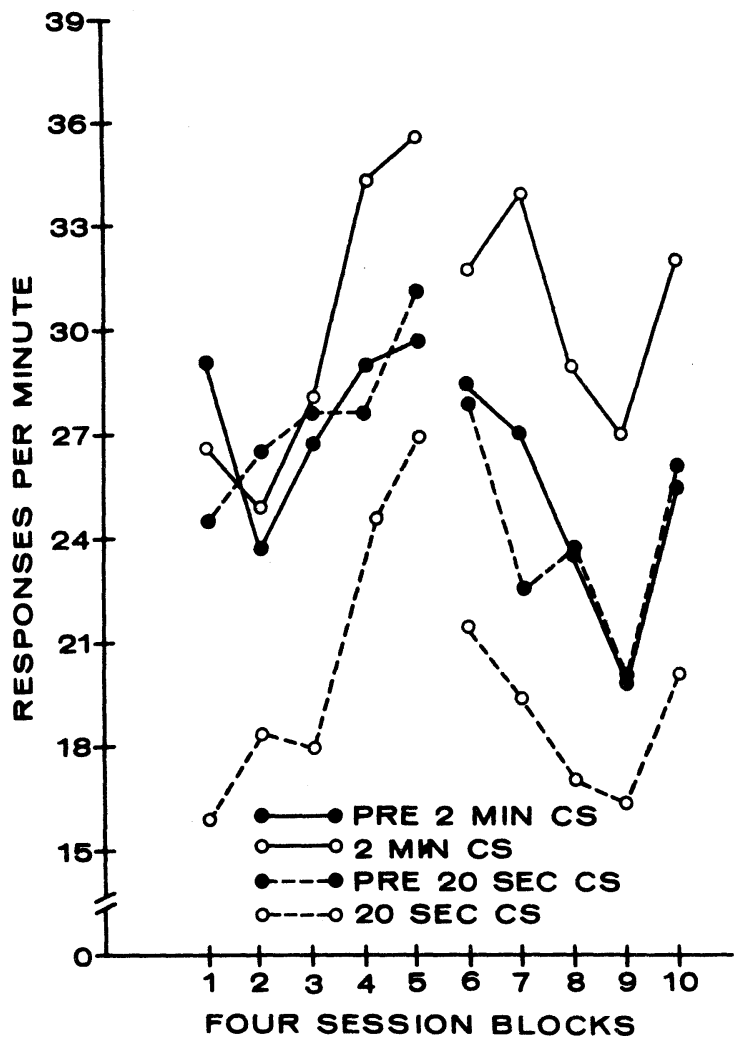

Figure 2. Mean response rates during the pre-CS intervals and during the long and short CSs in each of the 10 blocks of sessions.

The failure to find significant differences in the last half of the experiment is explained by the fact that the increase in response rate during long CSs almost always overlapped with the decrease in response rate of the group on the adjacent schedule during the short CS. For example, the mean response rate of subjects in the VI 2-min group during the short CS was 24.8 responses/min while the response rate in the VI 4-min group during the long CS was 30.7 responses $/ \mathrm{min}$.

When mean rates during the combined pre-CS and CS intervals were considered, overall rates were significantly higher during the pre-CS/long-CS sequence than during the pre-CS/short-CS sequence. Table 1 shows, however, the difference in rate was confined almost entirely to the CS intervals. This effect was significant during both the first $(F=17.23$, $\mathrm{df}=1 / 12, \mathrm{p}<.01)$ and the last $(\mathrm{F}=11.5, \mathrm{df}=1 / 11$, $\mathrm{p}<.05$ ) five blocks of sessions.

In the last block of sessions, response rate during the first and last $20 \mathrm{sec}$ of the long CS was compared with response rate during the short CS. As Figure 2 shows, the mean pre-CS rates for long and short CSs were almost identical during this block of sessions. The figure also shows that mean rate during the short CS was 20.2 responses/min. Although not shown in Figure 2, mean rate during the first $20 \mathrm{sec}$ of the long CS was 28.1 responses/min. Thirteen of the 17 
Table 1

Mean Response Rates During Pre-CS and CS Intervals for the First and Last Five Blocks of Sessions

\begin{tabular}{|c|c|c|c|c|c|}
\hline \multirow[b]{3}{*}{ Schedule } & \multirow{3}{*}{$\begin{array}{c}\text { CS } \\
\text { Duration }\end{array}$} & \multicolumn{4}{|c|}{ Rate } \\
\hline & & \multicolumn{2}{|c|}{ First Five Blocks } & \multicolumn{2}{|c|}{ Last Five Blocks } \\
\hline & & pre-CS & $\mathrm{CS}$ & pre-CS & CS \\
\hline VI 2 & $\begin{array}{l}2 \mathrm{~min} \\
20 \mathrm{sec}\end{array}$ & $\begin{array}{l}40.5 \\
39.3\end{array}$ & $\begin{array}{l}42.3 \\
28.8\end{array}$ & $\begin{array}{l}37.6 \\
36.3\end{array}$ & $\begin{array}{l}44.9 \\
24.8\end{array}$ \\
\hline VI 4 & $\begin{array}{c}2 \mathrm{~min} \\
20 \mathrm{sec}\end{array}$ & $\begin{array}{l}27.6 \\
27.9\end{array}$ & $\begin{array}{l}31.8 \\
21.0\end{array}$ & $\begin{array}{l}23.1 \\
22.1\end{array}$ & $\begin{array}{l}30.7 \\
18.4\end{array}$ \\
\hline VI 8 & $\begin{array}{l}2 \mathrm{~min} \\
20 \mathrm{sec}\end{array}$ & $\begin{array}{l}15.0 \\
15.0\end{array}$ & $\begin{array}{l}17.4 \\
12.9\end{array}$ & $\begin{array}{l}14.9 \\
14.9\end{array}$ & $\begin{array}{l}17.8 \\
11.2\end{array}$ \\
\hline
\end{tabular}

subjects had higher mean rates during the first $20 \mathrm{sec}$ of the long CS than during the short CS, which a sign test indicated to be significant at the .01 level. Mean rate during the last $20 \mathrm{sec}$ of the long CS (also not shown in figure) was 24.3 responses/min. Only 11 of the 17 subjects, however, had higher response rates during the last $20 \mathrm{sec}$ of the long CS than during the short CS, and a sign test showed that this difference was not significant.

\section{DISCUSSION}

The most important result of this experiment was the demonstration that conditioned enhancement and positive conditioned suppression could be brought under stimulus control within a single session. Both were shown to depend upon CS duration. Neither result could be accounted for by accidental juxtapositions of responses and UCSs since there was no reason why such accidental pairings should produce different results as a function of CS duration. Nor does it seem possible that enhancement should occur only if baseline response rates are low, as Mackintosh (1974) has suggested. In fact, the present results showed that both suppression and enhancement were the consequence of differences in response rates during the $\mathrm{CS}$ rather than shifting baseline rates.

The question still remains as to why such differences occur. Ever since Rescorla and Solomon (1967) predicted that a CSpositive UCS sequence would produce increases in the rate of a positively reinforced instrumental response, a two-factor interpretation has had no difficulty in explaining the development of conditioned enhancement. The basis of the suppression observed during the short CS, however, is still open to question. As mentioned previously, Hearst and Jenkins (1974) suggested that the reduction in response rate was actually the consequence of a competing response, namely, an orientation or approach to a localized stimulus which signaled the UCS. Schwartz's (1976) demonstration that pigeons pecked at a key-light CS preceding a food UCS, and thus produced suppression of instrumental responses, is a striking demonstration of this hypothesis. Unfortunately, once a diffuse CS such as a tone is used, the issue of localization becomes much more ambiguous. Although it is possible for a subject to locate a tone in space, there is no way to demon- strate that the subject is doing so unless it orients toward or approaches the speaker. Although observation revealed no such behavior by our subjects, significant conditioned suppression occurred when the tone was the short CS. Such a result suggests that sign-tracking may not be essential for the development of positive conditioned suppression. But, during the last five blocks of sessions, positive conditioned suppression was stronger when a light rather than a tone was the CS. Conditioned enhancement was less pronounced when a light rather than a tone was the CS. These effects are consistent with the idea that a strong orienting response occurs to a localized stimulus. But suppression or enhancement was an effect which was determined primarily by the duration of the CS. Whether suppression was entirely a sign-tracking response or depended upon some other type of anticipatory response to the UCS remains uncertain.

\section{REFERENCES}

Azrin, N. R.. \& Hake, D. R. Positive conditioned suppression: Conditioned suppression using reinforcers as the unconditioned stimuli. Journal of the Experimental Analysis of Behavior, 1969, 12, 167-173.

BRADY, J. V. Motivation-emotion factors and intracranial self-stimulation. In D. E. Sheer (Ed.), Electrical stimulation of the brain. Austin: University of Texas Press, 1961.

Hake, D. R., \& Powell, J. Positive reinforcement and suppression from the same occurrence of the unconditioned stimulus in a positive conditioned suppression procedure. Journal of the Experimental Analysis of Behavior, 1970, 14, 247-257.

HEARST, E., \& JENKINS, H. M. Sign tracking: The stimulus reinforcer relation and directed action. Austin: Psychonomic Society, 1974.

Mackintosh, N. J. The psychology of animal learning. New York: Academic Press, 1974.

Meltzer, D., \& Brahlek, J. A. Conditioned suppression and conditioned enhancement with the same positive UCS: An effect of CS duration. Journal of the Experimental Analysis of Behavior, 1970, 13, 67-74.

Meltzer, D., \& Hamm, R. J. Conditioned enhancement as a function of schedule of reinforcement. Bulletin of the Psychonomic Society, 1974, 3, 99-101. (a)

Meltzer. D., \& Hamm, R. J. Conditioned enhancement as a function of the percentage of CS-UCS pairings and CS duration. Bulletin of the Psychonomic Society. 1974, 4, 467-470. (b)

Myers, J. L. Fundamentals of experimental design. Boston: Allyn \& Bacon, 1972.

Rescorla, R. A., \& Solomon, R. L. Two-process learning theory: Relationship between Pavlovian conditioning and instrumental learning. Psychological Review, 1967, 64, 151-182.

Schwartz, B. Positive and negative conditioned suppression in the pigeon: Effects of the locus and modality of the CS. Learning and Motivation, 1976, 7, 86-100.

VAN Dyne, G. C. Conditioned suppression with a positive US in the rat. Journal of Comparative and Physiological Psychology, 1971, 77, 131-135.

(Received for publication September 26, 1977.) 